\title{
Combining Ability Analysis in Brinjal (Solanum melongena L.)
}

\author{
K. Hussain, S.H. Khan, Kouser Parveen, M.I. Mukhdoomi, G. Nazir, \\ B. Afroza, P.K. Singh and Z.A. Dar* \\ Division of Vegetable Science, Dryland Agriculture Research Station, Sher-e-Kashmir \\ University of Agricultural Sciences and Technology of Kashmir, Shalimar, \\ Srinagar - 190025 (J\&K), India \\ *Corresponding author
}

Keywords

Solanum

melongena L.,

Combining ability

effects, gca, sca,

Diallel.

Article Info

Accepted:

19 June 2017

Available Online:

10 July 2017

\section{A B S T R A C T}

Analysis of variance for combining ability revealed that variances due to gca and sca were significant for all the traits except for TSS that exhibited non-significant mean squares for gca and sca in environment $E_{1}$ only suggesting the importance of both types of variances in the inheritance of the traits. GCA and SCA also interacted significantly with the environments. The estimates of dominance variance were higher in magnitude than corresponding estimates of additive variance indicating the preponderance of non-additive gene action. None of the parents exhibited desirable gca effects for all the traits simultaneously. The overall ranking of genotypes revealed that the parents Pusa Purple Long, GBL-1, Arka Nidhi, SBW-11, Pusa Purple Cluster and Local Long exhibited significant desirable gca effects for most of the traits. None of the crosses exhibited significant desirable sca effects for all the traits simultaneously. However, the crosses Arka Kusumakar x Local Long, GBL-1 x Pusa Purple Long, Arka Nidhi x GOB-1, Arka Nidhi x Pusa Kranti and SBPL-27 x GOB-1 were found to be desirable for most of the traits.

\section{Introduction}

Brinjal (Solanum melongena L.) is one of the major and principle vegetable crops widely grown in both temperate and tropical regions of the globe. In India, it is cultivated over an area of 691.50 thousand hectares with an annual production of 12634.10 thousand tonnes and productivity of 18.30 tonnes hectare $^{-1}$ (Tiwari et al., 2012) while in Jammu and Kashmir, it occupies an area of 1.95 thousand hectares with an annual production of 38.13 thousand tonnes and productivity of 19.53 tonnes hectare ${ }^{-1}$ (Anonymous, 2011). In
$\mathrm{J} \& \mathrm{~K}$ state in general and Kashmir valley in particular only few cultivars of brinjal are available for commercial cultivation which are either poor yielders and/or susceptible to various biotic and abiotic stresses. In the face of increasing population, there is a need for increasing its production and productivity levels. Its diversification, off-season production, availability of diverse types and development of potential hybrids has resulted in change of local preferences. In view of changing local preference for colour, shape, 
taste, etc., it is not possible to have one common cultivar to suit different localities of a region and local preferences. It is, therefore, necessary to improve the locally preferred cultivars or develop new hybrid combinations for high yield, quality, consumer acceptability and meet diverse taste of locals. This necessitates the development of varieties/hybrids superior in yield and quality. However, due to continued selection, much of the variability has exhausted. In order to create variability and identify the desirable segregants, emphasis needs to be laid on hybridization between the parents. Before any such improvement programme is initiated through hybridization, there is a need to have knowledge on combining ability of different genotypes and the nature and magnitude of various genetic parameters operative in different characters. Several biometrical procedures are available for evaluation of parents and their crosses for their general and specific combining ability and to know the nature and magnitude of gene effects for expression of various metric traits. Diallel analysis as suggested (Griffing, 1956a,b) helps to generate basic information on nature of inheritance of traits and to assess the combining ability of parents. The information generated on general and specific combining ability can be effectively used to identify superior varieties to be used as parents for hybridization and also indicate cross combinations likely to yield desirable segregates.

\section{Materials and Methods}

The present investigation was carried out to study general and specific combining ability of parents and crosses, respectively. The experimental materials for the present investigation consisted of ten diverse parental lines viz., Pusa Purple Cluster, Arka Nidhi, Pusa Kranti, SBPL-27, Arka Kusumakar, SBW-11, GBL-1, GOB-1, Pusa Purple Long and Local Long crossed in a diallel fashion during Kharif 2011 and 45 cross combinations were generated as per method II and Model-I (Griffing, 1956a,b). The parents and $\mathrm{F}_{1}$ crosses were evaluated during Kharif 2012 in randomized complete block design with three replications at each of the three different locations viz., Vegetable Experimental Farm, Division of Vegetable Science, SKUAST-Kashmir, Shalimar $\left(\mathrm{E}_{1}\right)$, Regional Research Station and Faculty of Agriculture, Wadura $\left(\mathrm{E}_{2}\right)$ and Mountain Research Center for Field Crops, Khudwani, Anantnag $\left(E_{3}\right)$. The observations were recorded on days to first flowering, days to first fruit set, days to first fruit picking, plant height $(\mathrm{cm})$, plant spread $(\mathrm{cm})$, number of branches plant $^{-1}$, fruit length $(\mathrm{cm})$, fruit diameter $(\mathrm{cm})$, number of pickings plant $^{-1}$, number of fruits plant ${ }^{-1}$, average fruit weight $(\mathrm{g})$, fruit yield plant ${ }^{-1}(\mathrm{~kg})$, fruit yield $\left(\mathrm{q} \mathrm{ha}^{-1}\right)$, dry matter $(\%)$, TSS $\left({ }^{\circ}\right.$ Brix), vitamin $\mathrm{C}(\mathrm{mg}$ $\left.100 \mathrm{~g}^{-1}\right)$, anthocyanin (mg $100 \mathrm{~g}^{-1}$ ), total phenols (mg $100 \mathrm{~g}^{-1}$ ). The data thus generated, was subjected to standard statistical procedures to generate the results.

\section{Results and Discussion}

Analysis of variance for general and specific combining ability and their interactions with environment and estimates of components of variance are presented in table $1 \mathrm{a}$ and $1 \mathrm{~b}$. Analysis of variance for combining ability revealed that the mean squares due to environments were significant for all traits under consideration revealing that the environments chosen for the present investigation were diverse. Mean squares for general and specific combining ability were also significant for all traits under study in the pooled analysis. The mean squares due to gca $\mathrm{x}$ environment and sca $\mathrm{x}$ environment were found significant for all the traits. Variances due to sca $\left(\hat{\sigma}^{2} s\right)$ were higher in magnitude than the corresponding gca variances $\left(\hat{\sigma}^{2} g\right)$ for all the traits under consideration 
indicating the greater role of non-additive gene effects. The magnitude of dominance variance was found to be higher than the corresponding additive variance for all the traits again indicating the importance of nonadditive gene action. The ratio of additive genetic variance to dominance variance $\left(\hat{\sigma}^{2} A / \hat{\sigma}^{2} D\right)$ was less than unity for all the traits. Preponderance of non-additive gene action for various traits studied has also been reported (Chaudhary, 1999; Das and Barua, 2001; Singh et al., 2002a); Biradar et al., 2005; Suneetha and Kathiria, 2006; Quamruzzaman et al., 2007; Karibasava et al., 2010; Muniappan et al., 2010; Nalini et al., 2011; Rai and Asati, 2011; Hubaity and Teli, 2013; Raghvendra et al., 2014; Reddy and Patel, 2014; Sharif Uddin et al., 2015).

None of the parents possessed significant and desirable general combining ability effects for all the traits simultaneously. However, different parents were found to reveal desirable general combining ability for different trait (Table 2a and 2b).

Arka Nidhi, SBW-11 and GBL-1 showed significant negative gca effects for maturity traits viz., days to first flowering, days to first fruit set and days to first fruit picking in all the environments and pooled analysis except Arka Nidhi in days to first flowering. The desirable combiners were Local Long, Arka Nidhi and Arka Kusumkar for plant height; Arka Nidhi, SBPL-27, Arka Kusumaker, Pusa Purple Long and Local Long for plant spread; Arka Kusumaker, GBL-1 and Pusa Purple Long for number of branches plant ${ }^{-1}$; Arka Nidhi, Arka Kusumaker, GBL-1, Pusa Purple Long and Local Long for fruit length and Pusa Kranti and GOB-1 for fruit diameter. Desirable gca effects were also depicted by SBW-11, GOB-1, Pusa Purple Long and Local Long for average fruit weight; Pusa Purple Cluster, Arka Nidhi, SBPL-27, GBL-1 and Pusa Purple Long for number of fruits plant $^{-1}$ and Arka Kusumaker, SBW-11, GBL1 and PPL for number of pickings plant ${ }^{-1}$; Pusa Purple Cluster, GBL-1, PPL and L. Long for fruit yield plant ${ }^{-1}$. GBL-1 and GOB1 showed significant positive effect for dry matter content while most of the parent's viz., SBPL-27, Arka Kusumaker, SBW-11, GBL1, GOB-1 and Local Long exhibited nonsignificant gca effects for TSS in all individual environments and pooled analysis. Pusa Purple Long exhibited significant positive gca effect in all individual environments and pooled analysis where it exhibited non-significant gca effect; Pusa Purple Cluster exhibited significant positive gca effect in pooled analysis while nonsignificant gca effect. Arka Nidhi and Pusa Kranti were found to be good combiners for vitamin C; Arka Nidhi, Pusa Kranti, GBL-1, GOB-1 for anthocyanin and SBPL-27, Arka Kusumaker, SBW-11, GOB-1, Pusa Purple Long and Local Long were found to be good combiners with significant positive gca effect for total phenols in individual environments and data pooled over environments. In most of the cases, high general combining ability for the traits was associated with their high to average per se performance and poor general combining ability was associated with low per se performance.

None of the parents exhibited superior general combining ability for all the traits simultaneously. However, overall ranking of genotypes revealed that the parents PPL, GBL-1, A. Nidhi, SBW-11, PPC and L. Long exhibited significant desirable gca effects for most of the traits. Similar results were obtained (Chaudhary, 1999; Das and Barua, 2001; Singh et al., 2002a; Biradar et al., 2005; Suneetha and Kathiria, 2006; Quamruzzaman et al., 2007; Karibasava et al., 2010; Muniappan et al., 2010; Nalini et al., 2011; Rai and Asati, 2011; Hubaity and Teli, 2013; Raghvendra et al., 2014; Reddy and Patel, 2014; Sharif Uddin et al., 2015). 
Table.1a Analysis of variance for combining ability and estimates of components of variance for maturity and yield attributing traits in brinjal (Solanum melongena L.) (Pooled analysis)

\begin{tabular}{|c|c|c|c|c|c|c|c|c|c|}
\hline Source of variation & d.f & $\begin{array}{l}\text { Days to first } \\
\text { flowering }\end{array}$ & $\begin{array}{l}\text { Days to first } \\
\text { fruit set }\end{array}$ & $\begin{array}{l}\text { Days to first } \\
\text { fruit picking }\end{array}$ & $\begin{array}{l}\text { Plant height } \\
\text { (cm) }\end{array}$ & $\begin{array}{c}\text { Plant spread } \\
(\mathrm{cm})\end{array}$ & $\begin{array}{c}\text { Number of } \\
\text { branches plant } \\
1\end{array}$ & $\begin{array}{l}\text { Fruit length } \\
(\mathrm{cm})\end{array}$ & $\begin{array}{l}\text { Fruit diameter } \\
(\mathrm{cm})\end{array}$ \\
\hline Environments & 2 & $31811.76^{* * *}$ & $41069.03 * *$ & $76473.91^{* * *}$ & $20619.50 * *$ & $52702.14 * *$ & $5980.62 * *$ & $3365.56^{* *}$ & $489.51 * *$ \\
\hline gca & 9 & $8.07 * *$ & $5.02 * *$ & $5.10 * *$ & $47.05^{* *}$ & $27.18^{* *}$ & $3.87 * *$ & $14.86^{* *}$ & $1.09 * *$ \\
\hline sca & 45 & $9.09 * *$ & $9.97 * *$ & $9.75 * *$ & $12.08^{* *}$ & $30.05^{* *}$ & $1.91 * *$ & $2.25 * *$ & $0.29 * *$ \\
\hline gca $\mathrm{x}$ environments & 18 & $8.07 * *$ & $5.02 * *$ & $5.10 * *$ & $47.05^{* *}$ & $27.18^{* *}$ & $3.87 * *$ & $14.86^{* *}$ & $1.09 * *$ \\
\hline sca $\mathrm{x}$ environments & 90 & $9.09 * *$ & $9.97 * *$ & $9.75 * *$ & $12.08^{* *}$ & $30.05^{* *}$ & $1.91 * *$ & $2.25^{* *}$ & $0.29 * *$ \\
\hline Error & 154 & -- & -- & -- & -- & -- & -- & -- & -- \\
\hline Pooled & 324 & 0.16 & 0.08 & 0.08 & 0.04 & 0.06 & 0.06 & 0.02 & 0.01 \\
\hline$\hat{\sigma}^{2} g$ & - & 0.22 & 0.13 & 0.13 & 1.30 & 0.75 & 0.10 & 0.41 & 0.03 \\
\hline$\hat{\sigma}^{2} s$ & - & 2.97 & 3.29 & 3.22 & 4.01 & 9.99 & 0.61 & 0.74 & 0.09 \\
\hline$\hat{\sigma}^{2} A$ & - & 0.44 & 0.27 & 0.27 & 2.61 & 1.50 & 0.21 & 0.82 & 0.06 \\
\hline$\hat{\sigma}^{2} D$ & - & 2.97 & 3.29 & 3.22 & 4.01 & 9.99 & 0.61 & 0.74 & 0.09 \\
\hline$\hat{\sigma}^{2} A / \hat{\sigma}^{2} D$ & - & 0.15 & 0.08 & 0.08 & 0.65 & 0.15 & 0.34 & 1.11 & 0.67 \\
\hline
\end{tabular}

Table.1b Analysis of variance for combining ability and estimates of components of variance for maturity and Yield attributing traits in brinjal (Solanum melongena L.) (Pooled analysis)

\begin{tabular}{|c|c|c|c|c|c|c|c|c|c|c|c|}
\hline Source of variation & d.f & $\begin{array}{l}\text { Average fruit } \\
\text { weight }\end{array}$ & $\begin{array}{c}\text { Number of } \\
\text { fruits per } \\
\text { plant }\end{array}$ & $\begin{array}{c}\text { Number of } \\
\text { pickings }^{-1} \\
\text { plant }^{-1}\end{array}$ & $\begin{array}{c}\text { Fruit } \\
\text { yield } \\
\text { plant }^{-1}\end{array}$ & $\begin{array}{c}\text { Fruit yield } \\
\text { ha }^{-1}\end{array}$ & Dry matter & T.S.S & Vitamin C & Anthocyanin & $\begin{array}{c}\text { Total } \\
\text { phenols }\end{array}$ \\
\hline Environments & 2 & $126691.70^{* *}$ & $4888.71 * *$ & $947.77 * *$ & $32.26 * *$ & $7258.68 * *$ & $916.56 * *$ & $402.52 * *$ & $3992.74 * *$ & $19.94 * *$ & $219388.30^{* * *}$ \\
\hline gca & 9 & $181.63^{* *}$ & $12.06 * *$ & $0.13^{* *}$ & $0.06^{* *}$ & $15.22 * *$ & $0.21 * *$ & $0.13^{* *}$ & $1.49 * *$ & $0.09^{* *}$ & $263.03 * *$ \\
\hline sca & 45 & $198.08 * *$ & $7.78 * *$ & $0.079 * *$ & $0.08 * *$ & $19.74 * *$ & $0.36 * *$ & $0.21 * *$ & $0.55 * *$ & $0.07 * *$ & $82.88^{* *}$ \\
\hline gca $x$ environments & 18 & $181.63^{* *}$ & $12.06 * *$ & $0.13 * *$ & $0.06 * *$ & $15.22 * *$ & $0.21 * *$ & $0.13 * *$ & $1.49 * *$ & $0.09 * *$ & $263.03 * *$ \\
\hline sca $\mathrm{x}$ environments & 90 & $198.08 * *$ & $7.78 * *$ & $0.079 * *$ & $0.08 * *$ & $19.74 * *$ & $0.36^{* *}$ & $0.21^{* *}$ & $0.55 * *$ & $0.07 * *$ & $82.88^{* *}$ \\
\hline Error & 154 & -- & -- & -- & -- & -- & -- & -- & -- & -- & -- \\
\hline Pooled & 324 & 0.16 & 0.009 & 0.000 & 0.00 & 1.84 & 0.006 & 0.03 & 0.04 & 0.001 & 0.11 \\
\hline$\hat{\sigma}^{2} g$ & - & 5.04 & 0.33 & 0.004 & 0.002 & 226.46 & 0.006 & 0.003 & 0.04 & 0.003 & 7.30 \\
\hline$\hat{\sigma}^{2} s$ & - & 65.97 & 2.59 & 0.026 & 0.029 & 3523.15 & 0.120 & 0.061 & 0.17 & 0.025 & 27.59 \\
\hline$\hat{\sigma}^{2} A$ & - & 10.08 & 0.67 & 0.007 & 0.004 & 452.92 & 0.012 & 0.005 & 0.08 & 0.005 & 14.60 \\
\hline$\hat{\sigma}^{2} D$ & - & 65.97 & 2.59 & 0.026 & 0.029 & 3523.15 & 0.120 & 0.061 & 0.17 & 0.025 & 27.59 \\
\hline$\hat{\sigma}^{2} A / \hat{\sigma}^{2} D$ & - & 0.15 & 0.26 & 0.27 & 0.14 & 0.13 & 0.10 & 0.08 & 0.47 & 0.20 & 0.53 \\
\hline
\end{tabular}

*, ** Significant at 5 and 1 per cent levels, respectively 
Table.2a General combining ability effects for maturity and yield attributing traits in brinjal (Solanum melongena L.)

\begin{tabular}{|c|c|c|c|c|c|c|c|c|}
\hline Parents & $\begin{array}{l}\text { Days to first } \\
\text { flowering }\end{array}$ & $\begin{array}{l}\text { Days to first } \\
\text { fruit set }\end{array}$ & $\begin{array}{l}\text { Days to first } \\
\text { fruit picking }\end{array}$ & $\begin{array}{l}\text { Plant height } \\
\text { (cm) }\end{array}$ & $\begin{array}{l}\text { Plant spread } \\
\text { (cm) }\end{array}$ & $\begin{array}{c}\text { Number of } \\
\text { branches }\end{array}$ & $\begin{array}{l}\text { Fruit length } \\
\text { (cm) }\end{array}$ & $\begin{array}{c}\text { Fruit } \\
\text { diameter }(\mathrm{cm})\end{array}$ \\
\hline PPC & $-0.944 * *$ & $0.374^{*}$ & -0.213 & $-1.096 * *$ & $-3.158 * *$ & $-2.034 * *$ & $-1.444 * *$ & $-0.227 * *$ \\
\hline A.Nidhi & 0.296 & $-0.580 * *$ & $-0.500 * *$ & $3.393 * *$ & $2.546 * *$ & $-0.912 * *$ & $1.733 * *$ & $-0.214 * *$ \\
\hline P.Kranti & $0.518^{*}$ & -0.043 & 0.018 & $-0.715 * *$ & $-4.056^{* *}$ & $0.269 *$ & 0.015 & $0.820 * *$ \\
\hline SBPL-27 & $0.759 * *$ & 0.161 & 0.205 & $-2.951 * *$ & $4.512 * *$ & $-0.309 *$ & $-1.798 * *$ & $-0.399 * *$ \\
\hline A.Kusumakar & $1.509 * *$ & $0.364 *$ & $0.425^{* *}$ & $1.556 * *$ & $0.621 * *$ & $1.147 * *$ & $1.151 * *$ & $-0.699 * *$ \\
\hline SBW-11 & $-2.212 * *$ & $-2.199 * *$ & $-2.175^{* *}$ & $-2.174 * *$ & $-2.032 * *$ & $0.229 *$ & $-2.156^{* *}$ & 0.063 \\
\hline GBL-1 & $-1.973 * *$ & $-0.672 * *$ & $-0.574 * *$ & $-0.219 *$ & 0.156 & $0.994 * *$ & $0.748 * *$ & $-0.339 * *$ \\
\hline GOB-1 & -0.009 & $0.347 *$ & $0.380 *$ & $-3.205 * *$ & $-0.683^{* *}$ & 0.135 & $-2.392 * *$ & $0.957 * *$ \\
\hline PPL & -0.232 & -0.043 & 0.037 & $-2.386^{* *}$ & $0.452 * *$ & $0.977 * *$ & $3.538 * *$ & -0.054 \\
\hline L. Long & $2.287 * *$ & $2.291 * *$ & $2.398 * *$ & $7.797 * *$ & $1.641 * *$ & $-0.495 * *$ & $0.605 * *$ & 0.094 \\
\hline $\mathrm{SE} \pm(\mathrm{g})$ & 0.190 & 0.130 & 0.130 & 0.100 & 0.120 & 0.110 & 0.070 & 0.050 \\
\hline $\mathrm{SE} \pm\left(\mathrm{g}_{\mathrm{i}}-\mathrm{g}_{\mathrm{j}}\right)$ & 0.280 & 0.200 & 0.200 & 0.340 & 0.180 & 0.170 & 0.110 & 0.080 \\
\hline
\end{tabular}

*,** Significant at 5 and 1 per cent levels, respectively

Table.2b General combining ability effects for maturity and yield attributing traits in brinjal (Solanum melongena L.)

\begin{tabular}{|c|c|c|c|c|c|c|c|c|c|c|}
\hline Parents & $\begin{array}{c}\text { Average } \\
\text { fruit } \\
\text { weight (g) } \\
\end{array}$ & $\begin{array}{l}\text { Number of } \\
\text { fruits plant }\end{array}$ & $\begin{array}{c}\text { Number of } \\
\text { pickings } \\
\text { plant }^{-1}\end{array}$ & $\begin{array}{c}\text { Fruit yield } \\
\text { plant }^{-1}\end{array}$ & $\begin{array}{c}\text { Fruit yield } \\
\text { ha }^{-1}\end{array}$ & $\begin{array}{c}\text { Dry } \\
\text { matter }\end{array}$ & T.S.S & $\begin{array}{c}\text { Vitamin } \\
\mathrm{C}\end{array}$ & Anthocyanin & $\begin{array}{c}\text { Total } \\
\text { phenols }\end{array}$ \\
\hline PPC & $-3.711 * *$ & $2.047 * *$ & $-0.202 * *$ & $0.140 * *$ & $48.782 * *$ & $-0.344 * *$ & $0.208^{*}$ & $-0.362 * *$ & $-0.141 * *$ & $-16.569 * *$ \\
\hline A.Nidhi & $-5.743 * *$ & $0.562 * *$ & $-0.340 * *$ & $-0.055^{* *}$ & $-18.709 * *$ & $-0.246^{* *}$ & $-0.415^{* *}$ & $0.820^{* *}$ & $0.162 * *$ & $-11.872 * *$ \\
\hline P.Kranti & $-3.981 * *$ & $-1.455^{* *}$ & $-0.064 * *$ & $-0.152 * *$ & $-52.997 * *$ & $-0.197 * *$ & 0.069 & $1.089 * *$ & $0.153 * *$ & $-1.904 * *$ \\
\hline SBPL-27 & $-4.167 * *$ & $0.146^{* *}$ & 0.003 & $-0.102 * *$ & $-34.919 * *$ & $-0.162 * *$ & 0.070 & $0.524 * *$ & $-0.143^{* *}$ & $2.314 * *$ \\
\hline A.Kusumakar & $-7.377 * *$ & $-0.432 * *$ & $0.130 * *$ & $-0.175^{* *}$ & $-60.618 * *$ & $0.180 * *$ & 0.002 & $-0.473 * *$ & $-0.283^{* *}$ & $3.652 * *$ \\
\hline SBW-11 & $6.147 * *$ & $-1.929 * *$ & $0.085 * *$ & $-0.057 * *$ & $-19.976 * *$ & $0.248 * *$ & 0.012 & -0.194 & $-0.107 * *$ & $8.836^{* *}$ \\
\hline GBL-1 & $-3.427 * *$ & $2.949 * *$ & $0.327 * *$ & $0.163 * *$ & $56.766^{* *}$ & 0.012 & -0.032 & $-0.281 *$ & $0.128 * *$ & $1.391 * *$ \\
\hline GOB-1 & $9.332 * *$ & $-2.218 * *$ & $0.021 * *$ & $-0.023 * *$ & $-8.185^{*}$ & -0.015 & -0.060 & -0.128 & $0.102 * *$ & $2.598 * *$ \\
\hline PPL & $1.262 * *$ & $1.372 * *$ & $0.076 * *$ & $0.158 * *$ & $54.671 * *$ & $0.219 * *$ & $0.236^{*}$ & $-0.851 * *$ & $0.112 * *$ & $5.272 * *$ \\
\hline L. Long & $11.665^{* *}$ & $-1.042 * *$ & $-0.035 * *$ & $0.101 * *$ & $35.184 * *$ & $0.305 * *$ & -0.090 & -0.143 & 0.018 & $6.283 * *$ \\
\hline $\mathrm{SE} \pm(\mathrm{g})$ & 0.190 & 0.045 & 0.004 & 0.002 & 2.160 & 0.035 & 0.080 & 0.099 & 0.011 & 0.150 \\
\hline $\mathrm{SE} \pm\left(\mathrm{g}_{\mathrm{i}}-\mathrm{g}_{\mathrm{j}}\right)$ & 0.280 & 0.220 & 0.006 & 0.003 & 3.180 & 0.053 & 0.130 & 0.140 & 0.016 & 0.230 \\
\hline
\end{tabular}

$*, * *$ Significant at 5 and 1 per cent levels, respectively 
Table.3a Specific combining ability effects for maturity and yield attributing traits in brinjal (Solanum melongena L.)

\begin{tabular}{|c|c|c|c|c|c|c|c|c|}
\hline Crosses & $\begin{array}{l}\text { Days to first } \\
\text { flowering }\end{array}$ & $\begin{array}{c}\text { Days to first fruit } \\
\text { set }\end{array}$ & $\begin{array}{l}\text { Days to first fruit } \\
\text { picking }\end{array}$ & Plant height $(\mathbf{c m})$ & Plant spread $(\mathbf{c m})$ & $\begin{array}{c}\begin{array}{c}\text { Number of } \\
\text { branches per } \\
\text { plant }\end{array} \\
\end{array}$ & Fruit length $(\mathbf{c m})$ & $\begin{array}{l}\text { Fruit diameter } \\
\quad(\mathbf{c m})\end{array}$ \\
\hline PPC x A.Nidhi & $-2.454 * *$ & $-3.677 * *$ & $-2.986^{* * *}$ & $6.717^{* *}$ & 0.306 & -0.295 & $-2.838^{* *}$ & $1.575^{* * *}$ \\
\hline PPC x P. Kranti & 0.434 & -0.218 & 0.386 & $-5.996 * *$ & $5.185^{* *}$ & 0.671 & $3.289 * *$ & $-0.616^{* *}$ \\
\hline PPC x SBPL-27 & $4.863^{* *}$ & $3.359^{* *}$ & $3.98 * *$ & $2.614 * *$ & $-2.36 * *$ & $2.258^{* * *}$ & $1.576^{* *}$ & 0.066 \\
\hline PPC x A.Kusumakar & $-4.221 * *$ & $-6.288 * *$ & $-5.688 * *$ & $-11.173 * *$ & $-7.569 * *$ & $3.679 * *$ & -0.050 & -0.178 \\
\hline PPC x SBW-11 & $-2.052 * *$ & $-3.501 * *$ & $-2.864 * *$ & $6.823 * *$ & $21.117 * *$ & $-1.323 * *$ & $1.204 * *$ & 0.301 \\
\hline PPC x GBL-1 & $1.481 *$ & $-1.588^{* *}$ & -1.021 & $1.945 * *$ & $1.396^{* *}$ & $3.065 * *$ & $-0.606 *$ & -0.047 \\
\hline PPC x GOB-1 & $5.631 * *$ & $3.283 * *$ & $3.915^{* *}$ & 0.338 & -0.018 & $-2.395 * *$ & $0.760 *$ & $0.577 * *$ \\
\hline PPC x PPL & $9.074 * *$ & $8.226^{* *}$ & $8.924 * *$ & -0.194 & $0.931 *$ & 0.052 & $-4.000 * *$ & $1.241 * *$ \\
\hline PPC x L. Long & $-4.555^{* *}$ & $-6.992 * *$ & $-6.547 * *$ & $-2.16^{* *}$ & $-6.055^{* *}$ & $-3.479 * *$ & -0.477 & $-0.947 * *$ \\
\hline A.Nidhi x P. Kranti & -0.137 & 0.293 & 0.116 & $-0.938 *$ & $-5.79 * *$ & $-1.308^{* *}$ & $-1.241 * *$ & $-1.763^{* *}$ \\
\hline A.Nidhi x SBPL-27 & $-3.158 * *$ & $-2.134 * *$ & $-2.067 * *$ & $-6.748 * *$ & $-13.798 * *$ & $-1.217 * *$ & $2.713^{* *}$ & $-0.574 * *$ \\
\hline A.Nidhi x A.Kusumakar & $6.095^{* *}$ & $7.999 * *$ & $7.933 * *$ & $2.605 * *$ & $-6.257 * *$ & $1.583 * *$ & $2.077 * *$ & -0.184 \\
\hline A.Nidhi x SBW-11 & -0.743 & -0.441 & -0.797 & $-2.129 * *$ & $2.196 * *$ & $3.845 * *$ & $-0.816 * *$ & -0.056 \\
\hline A.Nidhi x GBL-1 & -0.979 & $-2.631 * *$ & $-2.624 * *$ & $3.976 * *$ & $-1.385^{* *}$ & $-2.024 * *$ & $1.507 * *$ & $-0.68 * *$ \\
\hline A.Nidhi x GOB-1 & $-4.056^{* *}$ & $-4.32 * *$ & $-4.242 * *$ & $2.406 * *$ & $1.477^{* *}$ & $2.349 * *$ & $3.443 * *$ & $1.614 * *$ \\
\hline A.Nidhi x PPL & $7.057^{* *}$ & $6.293 * *$ & $5.877^{* *}$ & 0.630 & $5.016^{* *}$ & 0.773 & $-4.350 * *$ & $1.878 * *$ \\
\hline A.Nidhi x L. Long & $5.538 * *$ & $6.289 * *$ & $6.626^{* *}$ & $14.251^{* *}$ & $9.574 * *$ & 0.302 & -0.286 & $-1.00^{* *}$ \\
\hline P.Kranti x SBPL-27 & $2.4 * *$ & $3.439^{* *}$ & $2.861 * *$ & -0.538 & -0.739 & $2.702 * *$ & $-2.756^{* *}$ & $1.939 * *$ \\
\hline P.Kranti x A.Kusumakar & $-4.573 * *$ & $-4.875^{* *}$ & $-4.586^{* *}$ & $3.669 * *$ & $-6.458 * *$ & 0.406 & 0.361 & $-0.428 *$ \\
\hline P.Kranti x SBW-11 & $5.815 * *$ & $6.245^{* *}$ & $6.238 * *$ & $3.255^{* * *}$ & $2.332 * *$ & -0.535 & $1.415^{* *}$ & $0.687 * *$ \\
\hline P.Kranti x GBL-1 & $2.799 * *$ & $3.275 * *$ & $3.414 * *$ & 0.184 & $-10.196 * *$ & $2.479 * *$ & $-2.715 * *$ & $1.786 * *$ \\
\hline P.Kranti x GOB-1 & $3.169 * *$ & $4.369 * *$ & $4.24 * *$ & $-1.07 * *$ & $2.776 * *$ & $2.608 * *$ & 0.238 & 0.2 \\
\hline P.Kranti x PPL & $-3.945 * *$ & $-5.241 * *$ & $-5.308 * *$ & $3.631 * *$ & $4.635^{* *}$ & $1.826 * *$ & $-1.826 * *$ & $-1.099 * *$ \\
\hline P.Kranti x L. Long & $4.536^{* *}$ & $4.978 * *$ & $4.998 * *$ & $2.552 * *$ & $3.586^{* *}$ & $2.088 * *$ & $1.788^{* *}$ & $0.216^{* *}$ \\
\hline SBPL-27 x A.Kusumakar & $-2.038 * *$ & -0.412 & -0.659 & $1.778 * *$ & $-2.949 * *$ & $1.267 * *$ & $-6.392 * *$ & $1.007 * *$ \\
\hline SBPL-27 x SBW-11 & $9.464 * *$ & $9.489 * *$ & $9.385^{* *}$ & $4.688^{* *}$ & $-1.393 * *$ & $-2.091 * *$ & $1.025^{* *}$ & $0.842 * *$ \\
\hline SBPL-27 x GBL-1 & $-1.889 *$ & $-3.708 * *$ & $-3.769 * *$ & $-0.977 *$ & $6.132 * *$ & $-2.623^{* *}$ & $-0.715 *$ & $-0.735^{* *}$ \\
\hline SBPL-27 x GOB-1 & $-5.296 * *$ & $-6.504 * *$ & $-6.616^{* *}$ & -0.514 & $-14.712 * *$ & $2.123 * *$ & $2.161 * *$ & -0.295 \\
\hline SBPL-27 x PPL & $-3.963 * *$ & $-3.784 * *$ & $-3.604 * *$ & $-15.939 * *$ & $26.197 * *$ & $1.517 * *$ & -0.212 & -0.16 \\
\hline SBPL-27 x L. Long & $2.851 * *$ & $1.331 *$ & $1.365 * *$ & $-0.702 *$ & $-16.285^{* *}$ & $-3.161 * *$ & 0.241 & $-0.852 * *$ \\
\hline A.Kusumakar x SBW-11 & $-2.953 * *$ & $-3.052 * *$ & $-2.835 * *$ & $-4.953 * *$ & $-8.319 * *$ & -0.537 & $4.279 * *$ & $-0.512 *$ \\
\hline A.Kusumakar x GBL-1 & -0.526 & $-1.689^{* *}$ & $-1.993 * *$ & 0.439 & $-2.74 * *$ & $-2.269 * *$ & $0.542 *$ & -0.576 ** \\
\hline A.Kusumakar x GOB-1 & -0.379 & 0.516 & 0.830 & $2.029 * *$ & $-1.198 * *$ & 0.667 & $-1.752 * *$ & $-0.429 *$ \\
\hline A.Kusumakar x PPL & $6.62 * *$ & $6.685^{* *}$ & $6.509^{* *}$ & $9.937 * *$ & $-1.955 * *$ & 0.011 & 0.275 & $0.479 *$ \\
\hline A.Kusumakar x L. Long & $-7.119 * *$ & $-9.872 * *$ & $-9.966 * *$ & $-1.233 * *$ & $29.555 * *$ & -0.257 & $0.895^{* *}$ & 0.147 \\
\hline SBW-11 x GBL-1 & 0.196 & 8.545 & $8.497 * *$ & $-1.111 * *$ & $3.306 * *$ & $1.893 * *$ & 0.093 & $-1.328^{* *}$ \\
\hline SBW-11 x GOB-1 & $-4.434 * *$ & $-6.031 * *$ & $-6.233 * *$ & $-2.235 * *$ & $-7.048 * *$ & $-3.902 * *$ & $-0.878 * *$ & 0.146 \\
\hline SBW-11 x PPL & $-4.101 * *$ & $-5.865 * *$ & $-5.781 * *$ & $-9.35 * *$ & $-10.692 * *$ & -0.224 & $-3.121 * *$ & $-0.846 * *$ \\
\hline SBW-11 x L. Long & $-1.844 *$ & $-3.086 * *$ & $-3.142 * *$ & -0.196 & $3.305^{* *}$ & $3.952 * *$ & $-0.878 * *$ & $0.506^{*}$ \\
\hline GBL-1 x GOB-1 & $-2.01 * *$ & $-5.338^{* *}$ & $-5.391 * *$ & $10.73 * *$ & $5.664 * *$ & $1.93 * *$ & 0.012 & $-0.724 * *$ \\
\hline GBL-1 x PPL & $4.212 * *$ & $-1.282^{*}$ & $-1.162 *$ & $-4.911 * *$ & $-4.237 * *$ & $3.088 * *$ & $8.319^{* *}$ & $-0.813^{* *}$ \\
\hline GBL-1 x L. Long & $-4.97 * *$ & $-7.612 * *$ & $-7.743 * *$ & $2.002 * *$ & 0.63 & 0.473 & 0.386 & $1.368 * *$ \\
\hline GOB-1 x PPL & $-14.638 * *$ & $-4.521 * *$ & $-4.559 * *$ & $5.115^{* *}$ & $4.662 * *$ & $-2.007 * *$ & $-1.315^{* *}$ & $-0.809^{* *}$ \\
\hline GOB-1 x L. Long & $5.733 * *$ & $4.812 * *$ & $4.527 * *$ & $-8.151 * *$ & 0.116 & $1.309 * *$ & $-1.585 * *$ & $-0.805 * *$ \\
\hline PPL x L. Long & $5.402 * *$ & $3.311 * *$ & $3.092 * *$ & $4.203 * *$ & $-14.538 * *$ & 0.747 & $-2.235 * *$ & $0.366 * *$ \\
\hline $\mathrm{SE} \pm\left(\mathrm{S}_{\mathrm{ij}}\right)$ & 0.63 & 0.46 & 0.46 & 0.34 & 0.41 & 0.39 & 0.26 & 0.19 \\
\hline$S E \pm\left(S_{i j}-S_{i k}\right)$ & 0.94 & 0.68 & 0.68 & 0.5 & 0.6 & 0.58 & 0.39 & 0.28 \\
\hline
\end{tabular}


Table.3b Specific combining ability effects for maturity and yield attributing traits in brinjal (Solanum melongena L.)

\begin{tabular}{|c|c|c|c|c|c|c|c|c|c|c|}
\hline Crosses & $\begin{array}{l}\text { Average fruit } \\
\text { weight (g) }\end{array}$ & $\begin{array}{l}\text { Number of } \\
\text { fruits plant }^{-1}\end{array}$ & $\begin{array}{l}\text { Number of } \\
\text { pickings plant }\end{array}$ & $\begin{array}{l}\text { Fruit yield } \\
\text { plant }^{-1}\end{array}$ & Fruit yield ha $^{-1}$ & Dry matter & T.S.S & Vitamin C & Anthocyanin & Total phenols \\
\hline PPC x A.Nidhi & 0.295 & $-1.279^{* * *}$ & $0.682 * *$ & $-0.109 * *$ & $-37.359 * *$ & $-0.291 *$ & -0.132 & $-1.062^{* *}$ & $-0.114 *$ & $9.322 * *$ \\
\hline PPC x P. Kranti & $20.643 * *$ & $1.018^{* *}$ & $-0.924 * *$ & $0.408^{* *}$ & $141.566 * *$ & -0.163 & $0.691 *$ & 0.492 & $-0.432 * *$ & $-2.349 * *$ \\
\hline PPC x SBPL-27 & $-1.317 * *$ & $1.22 * *$ & $-0.321 * *$ & $0.091 * *$ & $32.475 * *$ & $0.522 * *$ & -0.264 & -0.080 & -0.159 ** & $-10.517 * *$ \\
\hline PPC x A.Kusumakar & $-4.65 * *$ & $7.289 * *$ & $0.882 * *$ & $0.405^{* *}$ & $141.214 * *$ & $0.617 * *$ & -0.162 & $-2.843 * *$ & $0.431 * *$ & $-17.088^{* *}$ \\
\hline PPC x SBW-11 & $6.012 * *$ & $2.809 * *$ & $0.256^{* *}$ & $0.353 * *$ & $121.895 * *$ & $1.535 * *$ & 0.571 & 0.509 & $0.265^{* *}$ & $3.491 * *$ \\
\hline PPC x GBL-1 & $29.143 * *$ & $-3.816^{* *}$ & 0.015 & $0.203 * *$ & $71.490 * *$ & $0.731 * *$ & 0.158 & $-1.161 * *$ & 0.013 & 8.126 ** \\
\hline PPC x GOB-1 & $11.83 * *$ & $2.761 * *$ & $-0.339 * *$ & $0.459 * *$ & $158.974 * *$ & $-2.635^{* *}$ & 0.412 & 0.922 & $0.779 * *$ & $5.052 * *$ \\
\hline PPC x PPL & $6.343 * *$ & $-1.539 * *$ & $-0.394 * *$ & $-0.042 * *$ & $-15.375^{* *}$ & $0.568 * *$ & $-1.021^{* *}$ & 0.542 & 0.049 & $15.765 * *$ \\
\hline PPC x L. Long & 0.408 & $2.005^{* *}$ & $0.377 * *$ & $0.192 * *$ & $66.312 * *$ & $1.682 * *$ & $-1.114 * *$ & 0.584 & $-0.354 * *$ & $-7.066 * *$ \\
\hline A.Nidhi x P. Kranti & $26.124 * *$ & $3.144 * *$ & $-0.786^{* *}$ & $0.723^{* *}$ & $251.877 * *$ & $1.972 * *$ & -0.062 & $2.210 * *$ & -0.064 & -0.692 \\
\hline A.Nidhi x SBPL-27 & 0.947 & $2.052 * *$ & $-0.183 * *$ & $0.243 * *$ & $83.763 * *$ & $-0.373 *$ & -0.187 & $-1.198 * *$ & $-0.102 *$ & $-12.811 * *$ \\
\hline A.Nidhi x A.Kusumakar & $23.534 * *$ & $-8.329 * *$ & $-0.650 * *$ & $-0.337 * *$ & $-116.815^{* *}$ & $0.775 * *$ & $-0.672 *$ & -0.241 & $-0.418^{* *}$ & $-1.605^{* *}$ \\
\hline A.Nidhi x SBW-11 & $-6.244 * *$ & $2.541 * *$ & $0.650 * *$ & $0.135^{* *}$ & $46.469 * *$ & $0.260^{*}$ & 0.084 & -0.310 & $0.589 * *$ & $3.781 * *$ \\
\hline A.Nidhi x GBL-1 & $-18.426^{* *}$ & $-4.917 * *$ & $-0.177 * *$ & $-0.612 * *$ & $-212.616^{* *}$ & $0.743 * *$ & -0.152 & $2.991 * *$ & $-0.466 * *$ & $14.963^{* *}$ \\
\hline A.Nidhi x GOB-1 & $24.961 * *$ & $0.843 * *$ & $0.799 * *$ & $0.481 * *$ & $167.292 * *$ & $-0.320 *$ & $1.366 * *$ & $2.394 * *$ & $0.420^{* *}$ & $-19.292 * *$ \\
\hline A.Nidhi x PPL & $-2.839 * *$ & $-0.744 * *$ & $0.074 * *$ & $-0.103 * *$ & $-35.828 * *$ & $-0.777 * *$ & 0.206 & -0.213 & $1.010^{* *}$ & $9.268 * *$ \\
\hline A.Nidhi x L. Long & $16.915^{* *}$ & $1.507 * *$ & $-0.145^{* *}$ & $0.460^{* *}$ & $159.059 * *$ & $-0.897 * *$ & -0.171 & -0.771 & $0.517^{* *}$ & $6.387 * *$ \\
\hline P.Kranti x SBPL-27 & $-21.911 * *$ & $-0.931 * *$ & $0.201 * *$ & $-0.326 * *$ & $-113.519^{* *}$ & $-0.511^{* *}$ & 0.093 & 0.516 & $0.381 * *$ & $6.158 * *$ \\
\hline P.Kranti x A.Kusumakar & $19.419^{* *}$ & $2.601 * *$ & $0.074 * *$ & $0.557 * *$ & $192.143 * *$ & $0.970 * *$ & $1.991 * *$ & -0.373 & $0.484 * *$ & $5.937 * *$ \\
\hline P.Kranti x SBW-11 & $-35.762 * *$ & $1.765 * *$ & $0.119^{* *}$ & $-0.391 * *$ & $-134.856 * *$ & $-1.055^{* *}$ & $0.687 *$ & $1.281 * *$ & $0.228 * *$ & $10.793 * *$ \\
\hline P.Kranti x GBL-1 & $-8.864 * *$ & $-3.33 * *$ & $0.218 * *$ & $-0.368 * *$ & $-127.531 * *$ & -0.052 & $-1.342 * *$ & -0.232 & $0.434 * *$ & $3.081 * *$ \\
\hline P.Kranti x GOB-1 & $-24.594 * *$ & $2.523 * *$ & $0.183^{* *}$ & $-0.182 * *$ & $-63.123^{* *}$ & $1.332 * *$ & $-1.208^{* *}$ & -0.758 & $0.212 * *$ & $5.661 * *$ \\
\hline P.Kranti x PPL & $5.623^{* *}$ & $-2.367 * *$ & $0.468 * *$ & $-0.139 * *$ & $-48.066^{* *}$ & $-0.769 * *$ & -0.527 & -0.182 & $-0.827 * *$ & $9.607 * *$ \\
\hline P.Kranti x L. Long & $-35.256 * *$ & $0.414 * *$ & $-0.091 * *$ & $-0.483 * *$ & $-168.263 * *$ & $-0.922 * *$ & $-0.874 *$ & $1.696 * *$ & $0.593 * *$ & $11.316^{* *}$ \\
\hline SBPL-27 x A.Kusumakar & $-62.951 * *$ & $4.856 * *$ & 0.007 & $-0.866 * *$ & $-300.844 * *$ & $-0.832 * *$ & -0.027 & -0.082 & $-0.114 * *$ & $-1.168^{*}$ \\
\hline SBPL-27 x SBW-11 & $27.148^{* *}$ & $-2.903^{* *}$ & $0.165^{* *}$ & $0.139 * *$ & $48.097 * *$ & $-0.896 * *$ & $-0.947 *$ & -0.444 & $-0.353^{* *}$ & $1.295^{*}$ \\
\hline SBPL-27 x GBL-1 & $-13.701 * *$ & $5.798 * *$ & $0.151^{* *}$ & $0.158^{* *}$ & $55.401 * *$ & $-0.449 * *$ & -0.344 & $1.886^{* *}$ & $0.689 * *$ & $11.003^{* *}$ \\
\hline SBPL-27 x GOB-1 & $29.352 * *$ & $-3.168 * *$ & $0.146 * *$ & $0.101 * *$ & $35.396 * *$ & $0.644 * *$ & -0.352 & 0.408 & $-0.175 * *$ & $15.952 * *$ \\
\hline SBPL-27 x PPL & $30.486 * *$ & $-2.258 * *$ & $0.061 * *$ & $0.330 * *$ & 115.187 & $1.343 * *$ & $-0.695 *$ & -0.494 & $-0.285 * *$ & $17.398 * *$ \\
\hline SBPL-27 x L. Long & $16.963^{* *}$ & $-0.488^{* *}$ & $0.512^{* *}$ & $0.277 * *$ & $96.257^{* *}$ & $1.143 * *$ & $1.621 * *$ & 0.214 & -0.068 & $17.831^{* *}$ \\
\hline A.Kusumakar x SBW-11 & $-4.856 * *$ & $0.956 * *$ & $0.265^{* *}$ & $0.052 * *$ & 18.406 & $0.768 * *$ & 0.004 & 0.037 & $-0.199 * *$ & 0.647 \\
\hline A.Kusumakar x GBL-1 & $23.419 * *$ & $-7.979 * *$ & $-0.317 * *$ & $-0.278 * *$ & $-96.510 * *$ & $-0.709 * *$ & $1.301 * *$ & 0.690 & -0.094 & $13.162 * *$ \\
\hline A.Kusumakar x GOB-1 & $-15.051 * *$ & $-4.623 * *$ & $0.659 * *$ & $-0.498 * *$ & $-171.625^{* *}$ & $0.308 * *$ & 0.132 & 0.577 & $-0.229 * *$ & $11.318^{* *}$ \\
\hline A.Kusumakar x PPL & $-8.664 * *$ & $-1.129 * *$ & $-0.726 * *$ & $-0.209 * *$ & $-72.321 * *$ & -0.213 & $-0.981^{* *}$ & 0.947 & $0.368^{* *}$ & $16.491 * *$ \\
\hline A.Kusumakar x L. Long & $26.903 * *$ & $1.955^{* *}$ & $0.045^{* *}$ & $0.667 * *$ & $231.469 * *$ & $0.578 * *$ & $-0.674 *$ & $1.558^{* *}$ & 0.052 & $16.383^{* *}$ \\
\hline SBW-11 x GBL-1 & $7.164 * *$ & $-3.329^{* *}$ & $-0.272 * *$ & -0.130 & $-44.319^{* *}$ & $-1.211 * *$ & 0.144 & -0.245 & 0.013 & $-10.762 * *$ \\
\hline SBW-11 x GOB-1 & $12.045^{* *}$ & $1.907 * *$ & $-0.626 * *$ & $0.313 * *$ & $108.239 * *$ & $-0.993 * *$ & -0.194 & $-1.661 * *$ & -0.014 & $7.447 * *$ \\
\hline SBW-11 x PPL & $-3.339 * *$ & $-1.216^{* *}$ & $-0.021 * *$ & $-0.154 * *$ & $-53.897 * *$ & 0.086 & $-1.224 * *$ & 0.341 & $0.189 * *$ & $11.517 * *$ \\
\hline SBW-11 x L. Long & $15.526^{* *}$ & -0.082 & $-0.570 * *$ & $0.169^{* *}$ & $59.480 * *$ & $-1.447 * *$ & -0.087 & -0.577 & 0.073 & $14.076^{* *}$ \\
\hline GBL-1 x GOB-1 & $26.622 * *$ & $-4.561 * *$ & $-0.867 * *$ & $-0.010 * *$ & $-4.783 * *$ & $1.266^{* * *}$ & 0.003 & 0.372 & $0.434 * *$ & $17.415^{* *}$ \\
\hline GBL-1 x PPL & $1.602 * *$ & $22.289^{* *}$ & $1.078^{* *}$ & $1.899 * *$ & $658.584 * *$ & 0.208 & -0.100 & $0.708^{*}$ & $0.388 * *$ & $-13.838^{* * *}$ \\
\hline GBL-1 x L. Long & $4.677^{* *}$ & $1.153 * *$ & $0.518 * *$ & $0.272^{* *}$ & $93.934 * *$ & $0.856 * *$ & $0.603^{*}$ & 0.373 & $-0.109 *$ & $-1.169 * *$ \\
\hline GOB-1 x PPL & $22.069 * *$ & $-0.728 * *$ & $-0.617 * *$ & $0.252 * *$ & $86.819 * *$ & $1.139 * *$ & 0.224 & 0.089 & 0.070 & $-7.656 * *$ \\
\hline GOB-1 x L. Long & $-50.313 * *$ & $1.407 * *$ & $0.154 * *$ & $-0.628 * *$ & $-217.805^{* *}$ & $-0.794 * *$ & -0.213 & $-1.000 * *$ & -0.063 & $10.066 * *$ \\
\hline PPL x L. Long & $-35.913 * *$ & $-1.58 * *$ & $0.099 * *$ & $-0.679 * *$ & $-236.074 * *$ & $-1.191 * *$ & -0.012 & 0.393 & $0.691 * *$ & $11.026^{* *}$ \\
\hline $\mathbf{S E} \pm\left(\mathbf{S}_{\mathrm{ij}}\right)$ & 0.65 & 0.15 & 0.01 & 0.0065 & 1.94 & 0.12 & 0.29 & 0.33 & 0.037 & 0.53 \\
\hline$S E \pm\left(S_{i j}-S_{i k}\right)$ & 0.95 & 0.22 & 0.02 & 0.0096 & 3.18 & 0.15 & 0.43 & 0.49 & 0.055 & 0.78 \\
\hline
\end{tabular}


The specific combining ability of forty five crosses for different traits revealed that none of the $F_{1}$ crosses exhibited significant desirable effects for all the traits. Performance of some excellent crosses on the basis of SCA and per se performance together with the parental performance for each trait is given in table $3 \mathrm{a}$ and $3 \mathrm{~b}$. The crosses exhibiting significant desirable SCA effects (pooled over environments) included PPC x A. Nidhi, PPC $\mathrm{x}$ A. Kusmakar, PPC x SBW-11, PPC x L. Long, A. Nidhi x SBPL-27, A. Nidhi x GOB1, P. Kranti x A. Kusmakar, P.Kranti x PPL, SBPL-27 x A. Kusumakar, SBPL-27 x GBL1, SBPL-27 x GOB-1, SBPL-27 x PPL, A. Kusumakar x SBW-11, A. Kusumakar x L. Long, SBW-11 x GOB-1,SBW-11 x PPL, SBW-11 x L. Long, GBL-1 x GOB-1,GBL-1 $x$ L. Long and GOB-1 x PPL for days to first flowering; PPC $\mathrm{x}$ A. Nidhi, PPC $\mathrm{x}$ A. Kusumakar, PPC x SBW-11, PPC x L. Long, A. Nidhi x SBPL-27, A. Nidhi x GBL-1, A. Nidhi x GOB-1, P. Kranti x A. Kusumakar, P. Kranti x PPL, SBPL-27 x GBL-1, SBPL-27 $x$ GOB-1, $\quad$ SBPL-27 $x \quad$ PPL, A. Kusumakar x SBW-11, A. Kusmakar x GBL1, A. Kusumakar x L. Long, SBW-11 x GOB1, SBW-11 x PPL, SBW-11 x L. Long, GBL$1 \times$ GOB-1, GBL-1 x PPL, GOB-1 x PPL and GBL-1 $x$ L. Long for days to first fruit set; PPC x A. Nidhi, PPC x A. Kusumakar, PPC x SBW-11, PPC x GBL-1, PPC x L. Long, A. Nidhi $x$ SBPL-27, A. Nidhi $x$ GBL-1, A. Nidhi x GOB-1, P. Kranti x A. Kusumakar, P. Kranti x PPL, SBPL-27 x GBL-1, SBPL-27 x GOB-1, SBPL-27 x PPL, A. Kusumakar x SBW-11, A. Kusumakar $x$ GBL-1, A. Kusumakar x L. Long, SBW-11 x GOB-1, SBW-11 x PPL, SBW-11 x L. Long, GBL-1 x GOB-1, GBL-1 x PPL, GBL-1 x L. Long, GOB-1 x PPL for days to first fruit picking; PPC x SBPL-27, PPC x GBL-1, PPC x GOB1, A. Nidhi x A. Kusumakar, A. Nidhi x PPL, A. Nidhi x L. Long, P. Kranti x SBPL-27, P. Kranti x SBW-11, P. Kranti x GBL-1, P. Kranti x GOB-1, P. Kranti x L. Long, SBPL-
27 x SBW-11, SBPL-27 x L. Long, A. Kusumakar x PPL, SBW-11 x GBL-1,GOB-1 $x$ L. Long and PPL $x$ L. Long for plant height; PPC x P. Kranti, PPC x SBW-11, PPC x GBL-1, A. Nidhi $x$ SBW-11, A. Nidhi $x$ GOB-1, A.Nidhi x PPL, A. Nidhi x L. Long, P.Kranti $x$ SBW-11, P.Kranti x GOB-1, P.Kranti x PPL, P.Kranti x L. Long, SBPL-27 x GBL-1, SBPL-27 x PPL, A. Kusumakar x L. Long, SBW-11 x GBL-1, SBW-11 x L. Long, GBL-1 x GOB-1 and GOB-1 x PPL for plant spread; PPC $x$ SBPL-27, PPC $\times$ A. Kusumakar, PPC x GBL-1, A. Nidhi x A. Kusumakar, A. Nidhi x SBW-11, A.Nidhi $x$ GOB-1, A. Nidhi x PPL, P.Kranti x SBPL-27, P.Kranti x GBL-1, SBPL-27 x A. Kusumakar, P.Kranti x PPL, P. Kranti x L. Long, P.Kranti x GOB-1, SBPL-27 x GOB-1, SBPL-27 x PPL, SBW-11 x GBL-1, SBW-11 x L. Long, GBL-1 x GOB-1, GBL-1 x PPL and GOB-1 x L. Long for number of branches plant ${ }^{-1}$; PPC x P.Kranti, PPC x SBPL-27, PPC x SBW-11, PPC x GOB-1, A. Nidhi x SBPL-27, A. Nidhi x A. Kusumakar, A. Nidhi x GBL-1, A. Nidhi $\mathrm{x}$ GOB-1, P.Kranti $\mathrm{x}$ SBW-11, P.Kranti x L. Long, SBPL-27 x SBW-11, SBPL-27 x GOB-1, A. Kusmakar x SBW-11, A. Kusumakar x L. Long and GBL-1 x PPL for fruit length; PPC $x$ P. Kranti, PPC $x$ SBPL-27, PPC x SBW-11, PPC x GOB-1, A. Nidhi x SBPL-27, A. Nidhi x A. Kusumakar, A. Nidhi $x$ GBL-1, A. Nidhi $x$ GOB-1, P.Kranti x SBW-11, P.Kranti x L. Long, SBPL-27 x SBW-11, SBPL-27 x GOB-1, A. Kusumakar x SBW-11, A. Kusumakar x L. Long and GBL-1 x PPL for fruit diameter; PPC x P. Kranti, PPC x SBW-11, PPC x GBL-1, PPC x GOB-1, PPC x PPL, A. Nidhi x P. Kranti, A. Nidhi x A. Kusumakar, A. Nidhi x GOB-1, A. Nidhi x L. Long, P.Kranti x Arka Kusumakar, P. Kranti x PPL, SBPL27 x SBW-11, SBPL-27 x GOB-1, SBPL-27 x PPL, SBPL-27 x L. Long, Arka Kusumakar x GBL-1, A. Kusumakar x L. Long, SBW-11 x GBL-1, SBW-11 x GOB-1, SBW-11 x L. Long, GBL-1 x GOB-1, GBL-1 x L. Long 
and GOB-1 x PPL I for average fruit weight; PPC x SBPL-27, PPC x A. Kusumakar, PPC x SBW-11, PPC x GOB-1, PPC x L. Long, A. Nidhi x P. Kranti, A. Nidhi x SBPL-27, A. Nidhi x SBW-11, A. Nidhi x GOB-1, A. Nidhi x L. Long, P.Kranti x Arka.Kusumakar, P.Kranti x SBW-11, P.Kranti x GOB-1, P.Kranti $x$ L. Long, SBPL-27 $x$ A.Kusumakar, SBPL-27 $\mathrm{x}$ GBL-1, A.Kusumakar x L. Long, SBW-11 x GOB-1, GBL-1 x PPL, GOB-1 x L.Long for number of fruits plant ${ }^{-1}$; PPC $\mathrm{x}$ A.Nidhi, PPC $\mathrm{x}$ A.Kusumakar, PPC $x$ SBW-11, PPC $x$ L.Long, P.Kranti x GOB-1, P.Kranti x PPL, SBPL-27 x SBW-11, SBPL-27 x GOB-1, SBPL-27 x L.Long, A.Kusumakar x GOB-1 for number of pickings plant ${ }^{-1}$; PPC $\mathrm{x} P$. Kranti, PPC x A. Kusumakar, PPC x SBW11, PPC x GOB-1, A. Nidhi x P. Kranti, A. Nidhi x SBPL-27, A. Nidhi x SBW-11, A. Nidhi x GOB-1, A. Nidhi x L. Long, P.Kranti x A. Kusumakar, SBPL-27 x GOB-1, SBPL27 x PPL, SBPL-27 x L. Long, A. Kusumakar $x$ L. Long, SBW-11 x GOB-1, SBW-11 x L. Long, GBL-1 x PPL, GBL-1 x L. Long, GOB-1 x PPL for fruit yield; Pusa Purple Cluster x SBPL-27, Pusa Purple Cluster $x$ SBW-11, PPC x GBL-1, PPC x PPL, PPC x L. Long, A. Nidhi x P.Kranti, A. Nidhi x A. Kusumakar, A. Nidhi x GBL-1, P.Kranti x A. Kusumakar, P.Kranti x GOB-1, SBPL-27 x GOB-1, SBPL-27 x PPL, SBPL-27 x L.Long, A. Kusumakar x SBW-11, A.Kusumakar x L.Long, GBL-1 x GOB-1, GBL-1 x L.Long and GOB-1 x PPL for dry matter; A.Nidhi $x$ GOB-1, SBPL-27 $x \quad$ L.Long and A.Kusumakar x GBL-1 for TSS; A.Nidhi $\mathrm{x}$ P.Kranti, A.Nidhi x GBL-1, A.Nidhi x GOB1, P.Kranti x L.Long, SBPL-27 x GBL-1 and A.Kusumakar x L.Long for vitamin C; PPC x A.Kusumakar, Pusa Purple Cluster x SBW11, PPC $\mathrm{x}$ GOB-1, A.Nidhi $\mathrm{x}$ SBW-11, A.Nidhi x GOB-1, A.Nidhi x PPL, A.Nidhi $x$ L.Long, P.Kranti $x$ SBPL-27, P.Kranti $x$ A.Kusumakar, P.Kranti x GBL-1, P.Kranti x L.Long, SBPL-27 x GBL-1, A.Kusumakar x
PPL, GBL-1 x GOB-1, GBL-1 x PPL and PPL $x$ L.Long for anthocyanin and PPC $x$ A.Nidhi, Pusa Purple Cluster x GBL-1, PPC x GOB-1, Pusa Purple Cluster x Pusa Purple Long, A.Nidhi x SBW-11, A.Nidhi x GBL-1, A.Nidhi x GOB-1, A.Nidhi x PPL, A.Nidhi x L.Long, P.Kranti $x$ SBPL-27, P.Kranti $x$ A.Kusumakar, P.Kranti x SBW-11, P.Kranti x GBL-1, P.Kranti x GOB-1, P.Kranti x PPL, P.Kranti $x$ L. Long, SBPL-27 x GBL-1, SBPL-27 x GOB-1, SBPL-27 x PPL, SBPL$27 \mathrm{x}$ L. Long, A.Kusumakar $\mathrm{x}$ GBL-1, A.Kusumakar x GOB-1, A.Kusumakar x PPL, A.Kusumakar x L. Long, SBW-11 x GOB-1, SBW-11 x PPL, SBW-11 x L. Long, GBL-1 x GOB-1, GOB-1 x L. Long and PPL x L. Long for total phenols. None of the crosses exhibited significant desirable sca effects for all the traits simultaneously. However, the crosses A.Kusumakar x L.Long, GBL-1 x PPL, A.Nidhi x GOB-1, A.Nidhi x P.Kranti and SBPL-27 $x$ GOB-1 were found to be desirable for most of the traits. These desirable specific cross combinations resulted out of crosses between the parents with high $\mathrm{x}$ low followed by low $\mathrm{x}$ low, high $\mathrm{x}$ high and average $\mathrm{x}$ low general combining ability. Similar results for various characters in brinjal were also reported (Chaudhary, 1999; Das and Barua, 2001; Singh et al., 2002a; Biradar et al., 2005; Suneetha and Kathiria, 2006; Quamruzzaman et al., 2007; Karibasava et al., 2010; Muniappan et al., 2010; Nalini et al., 2011; Rai and Asati, 2011; Hubaity and Teli, 2013; Raghvendra et al., 2014; Reddy and Patel, 2014; Sharif Uddin et al., 2015).

The overall ranking of genotypes revealed that the parents Pusa Purple Long, GBL-1, Arka Nidhi, SBW-11, Pusa Purple Cluster and Local Long exhibited significant desirable gca effects for most of the traits. None of the crosses exhibited significant desirable sca effects for all the traits simultaneously. However, the crosses Arka 
Kusumakar x Local Long, GBL-1 x Pusa Purple Long, Arka Nidhi x GOB-1, Arka Nidhi x Pusa Kranti and SBPL-27 x GOB-1 were found to be desirable for most of the traits.

\section{References}

Anonymous. 2011. Department of Agriculture, Government of Jammu and Kashmir, p.1-2.

Aswani, R.C. and Khandelwal, R.C. 2005. Combining ability studies in brinjal. Indian J. Horticulture, 62(1): 37-40.

Biradar, A.B., Dumbre, A.D. and Navale, P.A. 2005. Combining ability studies in brinjal (Solanum melongena L.). J. Maharashtra Agri. Univ., 30(3): 342.

Bisht, G.S., Singh, M.C., Singh Major., Singh, S.K and Rai, M. 2006. Combining ability analysis in brinjal (Solanum melongena L.). Veg. Sci., 33(1): 68-70.

Chaudhary, D.R. 1999. Components of genetic variation in yield traits of brinjal (Solanum melongena L.). Himachal J. Agri. Res., 25(1-2): 55-61.

Das, G. and Barua, N.S. 2001. Heterosis and combining ability for yield and its components in brinjal. Annals of Agri. Res., 22(3): 399-403.

Griffing, J.B. 1956a. A generalized treatment on the use of diallel cross quantitative inheritance. Heredity, 10: 31-50.

Griffing, J.B. 1956 b. Concept of general and specific combining ability in relation to diallel crossing system. Australian J. Biol. Sci., 9: 463-93.

Hubaity, A.I. and Teli, J.A. 2013. Combining ability and heterosis in eggplant (Solanum melongena L.). Mesopotamia J. Agri., 41(1): 23-34.

Karibasava Naik, G.B., Sreenivasulu, S.J., Prashanth, R.P., Jayaprakashnarayan, M.B., Madalageri and Ravindra Mulge. 2010. Studies on genetic variability and its importance in brinjal (Solanum melongena L.). The Asian J. Horticulture, 4(2): 380-382.

Muniappan, S., Saravanan, K and Ramya, B. 2010. Studies on genetic divergence and variability for certain economic characters in eggplant (Solanum melongena L.). Electronic J. Plant Breeding, 1(4): 462-465.

Nalini, A. Dharwad, S., Patil, A and Salimath, P. M. 2011. Heterosis and combining ability analysis for productivity traits in brinjal (Solanum melongena L.). Karnataka J. Agri. Sci., 24(5): 622625.

Quamruzzaman, A.K.M., Rashid, M.A., Ahmad, S., Rahman M. $M$ and Sultana,N. A. 2007. Combining ability estimates in nine eggplant varieties. Pak. J. Scientific and Industrial Res., 50(1): 55-59.

Raghvendra, D., Arpita, D., Ojha, M.D., Bholanath, S., Ashish, R and Singh, K. 2014. Heterosis and combining ability studies for yield and yield attributing traits in brinjal (Solanum melongena L.). The Bioscan, 9(2): 889-894.

Rai, N. and Asati, B.S. 2011. Combining ability and gene action studies for fruit yield and yield contributing traits in brinjal (Solanum melongena L.). Indian J. Horticulture, 68(2): 212-215.

Reddy and Patel, A.I. 2014. Studies on gene action and combining ability for yield and other quantitative traits in brinjal (Solanum melongena L.). Trends in Biosci., 7(5): 381-383.

Sharuf Uddin, M., Rahman, M.M., Hossian, M.M and Khaleque Main, M.A. 2015. Combining ability of yield components in eggplant (Solanum melongena L.) during summer. Universal J. Plant Sci., 3(4): 59-66.

Singh, H.V., Singh, S. P., Singh Major, S., Singh, M., Singh, S. 2002a. Genetic analysis of quantitative traits in brinjal 
(Solanum melongena L.). Veg. Sci., 29(1): 84-86.

Suneetha,Y., Kathiria, K.B., Patel, J. S., Patel, N, B., Kathiria, P. K and Srinivas, T. 2006. Genetic studies in brinjal. Res. Crops, 7(3): 774-781.

Suneetha, Y. and Kathiria, K.B. 2006. Studies on combining ability for yield, quality and physiological characters in late summer brinjal. Int. J. Agri. Sci., 2(1): 193-197.
Suneetha, Y., Kathiria, K.B., Kathiria, P.K and Srinivas, T. 2008. Combining ability for yield and yield components in late summer brinjal. New Botanist, XXXV, 1-11.

Tiwari, R.K., Mistry, N.C., Singh, B and Gandhi, C.R.2012. All India area, production and productivity of brinjal. Indian Horticulture Database, p. 133.

\section{How to cite this article:}

Hussain, K., S.H. Khan, Kouser Parveen, M.I. Mukhdoomi, G. Nazir, B. Afroza, P.K. Singh and Dar, Z.A. 2017. Combining Ability Analysis in Brinjal (Solanum melongena L.). Int.J.Curr.Microbiol.App.Sci. 6(7): 1645-1655. doi: https://doi.org/10.20546/ijcmas.2017.607.199 